\title{
Reflections on a concurrent design methodology: a case study in wearable computer design
}

\author{
Susan Finger, John Stivoric, Cristina Amon, Levent Gursoz, Fritz Prinz, Daniel \\ Siewiorek, Asim Smailagic and Lee Weiss
}

\begin{abstract}
At Carnegie Mellon, we have designed and manufactured three generations of wearable, mobile computers. Each new generation of wearable computer has been designed within approximately one semester by an interdisciplinary design class taught at the Engineering Design Research Center (EDRC). Over the semesters that the course has been taught, an interdisciplinary concurrent design methodology has evolved. In this paper, we briefly present the design process for the Navigator, the third generation of wearable computers. We use this process to illustrate the needs of a multidisciplinary design team, to anticipate the needs of a distributcd design team using a novel manufacturing process, and to reflect on the interplay between the practice of design and the evolution of our design methods. Copyright (c) 1996 Elsevier Science Ltd
\end{abstract}

Keywords: design methodology, mobile computers, concurrent engineering

\section{INTRODUCTION}

At Carnegie Mellon University (Carnegie Mellon), we have designed and manufactured three generations of wearable, mobile computers: VuMan $1^{1}$, VuMan $2^{2}$ and Navigator ${ }^{3}$. We are currently designing VuMan 3 , a rugged, specialized computer for field maintenance, and Navigator 2, a general purpose, modular mobile computer with speech recognition and global positioning capabilities.

Mobile computers are novel devices that allow users to merge their information space with their workspace. Because mobile computers deal in information rather

Engineering Design Research Center, Carnegie Mellon University, Pittsburgh, PA 15213, USA

Paper received: 15 April 1994 than programs, they serve as tools in the user's envi ronment; however, unlike common tools such as pencils and reference books, mobile computers interact with the user and the environment.

The development time for each new generation of mobile computer is approximately one semester, since each computer is designed and prototyped by the students in an interdisciplinary design class that is offered through the Engineering Design Research Center at Carnegie Mellon. We have used this design course as a testbed for understanding how interdisciplinary teams work and interact. From our experience, we have developed a concurrent design methodology that is used in the class. Each generation provides a learning experience and experimental testbed enabling advancement towards the next generation.

To date, we have used conventional manufacturing technologies to prototype the wearable computers. In future generations, we will use a novel manufacturing process, Shape Deposition Manufacturing, which enables the manufacture of cmbcdded conformal electronics. Using Shape Deposition Manufacturing, the shape of the mobile computers will be able to conform to the human shape. Shape Deposition Manufacturing is an active research project, so we are working in an environment in which both the product and the process are undergoing continuous and rapid change. This requires that the design methodology be flexible and adaptable so that the tools and process can change as needed.

Currently, the entire design team for the mobile computers is located at Carnegie Mellon; however, for future generations of Navigator, we are planning to move toward distributed, networked design teams in which team members will be in different locations. By designing, manufacturing, and using our own tools where the tools may be the mobile computers or the design and analysis tools - we learn about design 
education and design practice, and we uncover new issues for design research.

\section{Wearable computers}

Wearable, mobile computers are the leading edge of a new generation of computer systems. These portable computers move with the user; they can track the user's motions in both time and space providing real-time information that can extend the user's knowledge and perception of the environment. The convergence of a variety of technologies makes this new mode of information processing possible. High-performance microprocessors can have low power and space requirements. Miniature head-worn displays weighing less than a few ounces are commercially available. Mobile communication technology enables users to access information anywhere. Global positioning systems allow the user's position to be sensed. Advances in speech recognition now enable a user to interact with the computer without a keyboard, allowing hands-free operation.

Mobile computers allow users greater interaction with the physical environment by providing real-time information directly relevant to the current state of the workspace. Support for augmented perception provides the user with a means of viewing details of the work environment which are otherwise invisible. For example, a maintenance worker can view what is behind a wall by displaying the appropriate blueprint and can record maintenance actions as they are made.

The first wearable computer designed and manufactured at Carnegie Mellon, the VuMan $1^{1}$, is shown in Figure 1. Over a 12-week period during the summer of 1991 in conjunction with a Carnegie-Bosch Institute continuing education course, we conceived, designed and manufactured this first generation wearable computer. VuMan 1 is a small portable computer weighing less than $1 \mathrm{~kg}$ for displaying construction blueprints.

The second mobile computer, VuMan 2, shown in Figure 2, developed during the fall quarter of 1992, is dedicated to campus navigation ${ }^{2}$. Compared to VuMan 1 , it was cheaper, lighter, simpler, and consumed less power. Composed of only five computer chips, VuMan 2 allows the user to select items from either a map. image database, or textual database using a hand-held. three button input device similar to a mouse.

The demonstration application for the third mobile computer, Navigator ${ }^{3}$, is also campus navigation. The Navigator shown in Figure 3, allows hands-off operation with a speaker-independent, 200 word vocabulary continuous speech recognition system for input and has a head-worn display for output. It provides wireless communication with a remote site, as well as global position sensing, so that information relevant to a location can be displayed. The first Navigator was designed and built in the spring of 1993 .

\section{Conformal electronics using shape deposition manufacturing}

The first generations of VuMan and Navigator used conventional manufacturing technology with the electronics being laid out on $2 \mathrm{D}$ printed circuit boards. surrounded by a housing. The necessity of designing

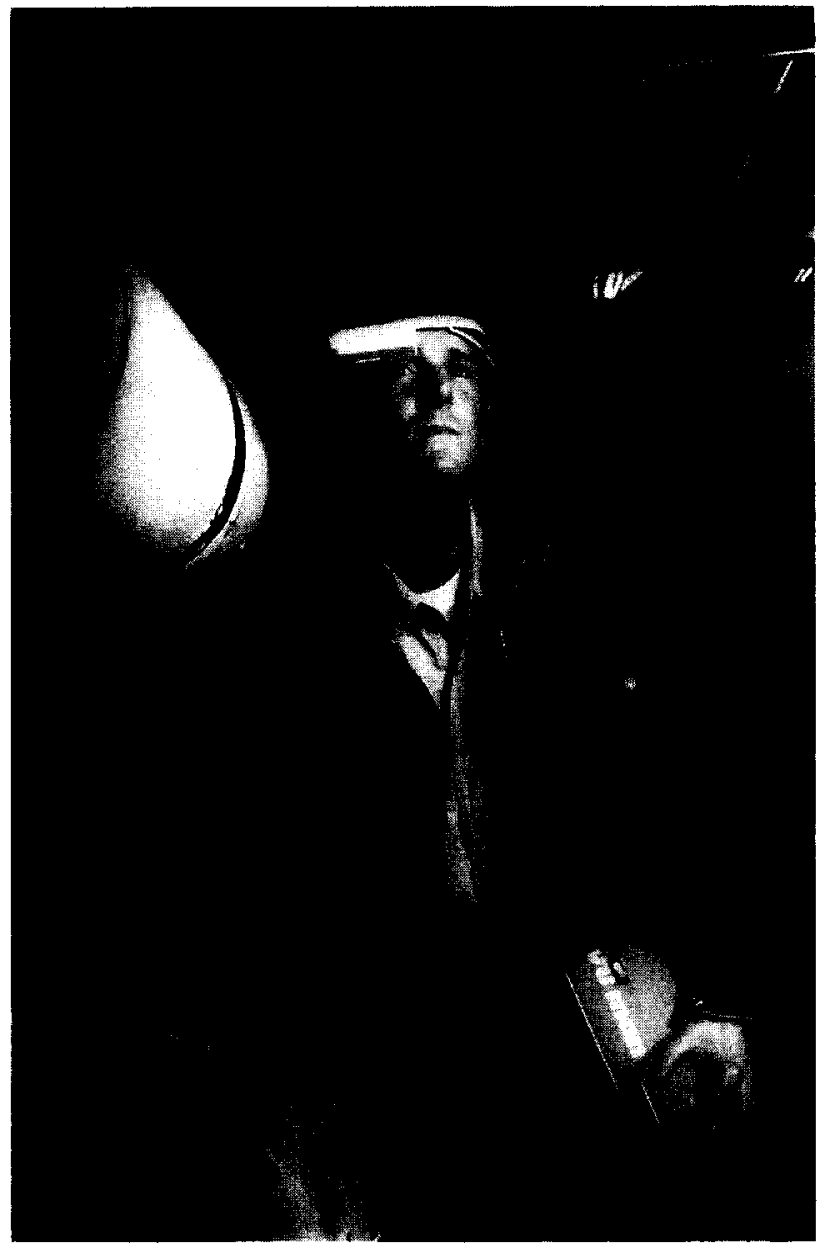

Figure 1 VuMan 1

around flat boards has limited the degree to which the Navigator can be worn as opposed to carried.

Future generations of wearable computers will take advantage of a new manufacturing process, called Shape Deposition, currently being developed at Carnegie Mel$l^{\prime}{ }^{4}$. Shape Deposition enables electronic components to be embedded within mechanical structures allowing for 3D layouts and closer packing of components.

Shape Deposition Manufacturing is a layered manufacturing process in which parts and assemblies are manufactured by successively depositing material in cross-sectional areas. Starting from a geometric model, the part is discretized into thin layers based on geometric as well as material criteria. The part is built by a vertical concatenation of two-and-a-half dimensional layers. Each layer undergoes a series of processes including material addition, stress relief, selective material removal, and surface preparation. The basic sequence of operations in the Shape Deposition process is shown in Figure 4. Currently, we are depositing steel and copper using the microcasting process. The deposition robot is equipped with a tool changing wrist, so materials such as plastics, wax, ceramics, glue, and plyurethane can be laid down using other robotic deposition processes. Additional processes, both manual and automated such as embedding prefabricated parts and testing the electronic circuits, can occur between repetitions of this loop.

In the Shape Deposition process, creating each layer requires several manufacturing subprocesses. One of 


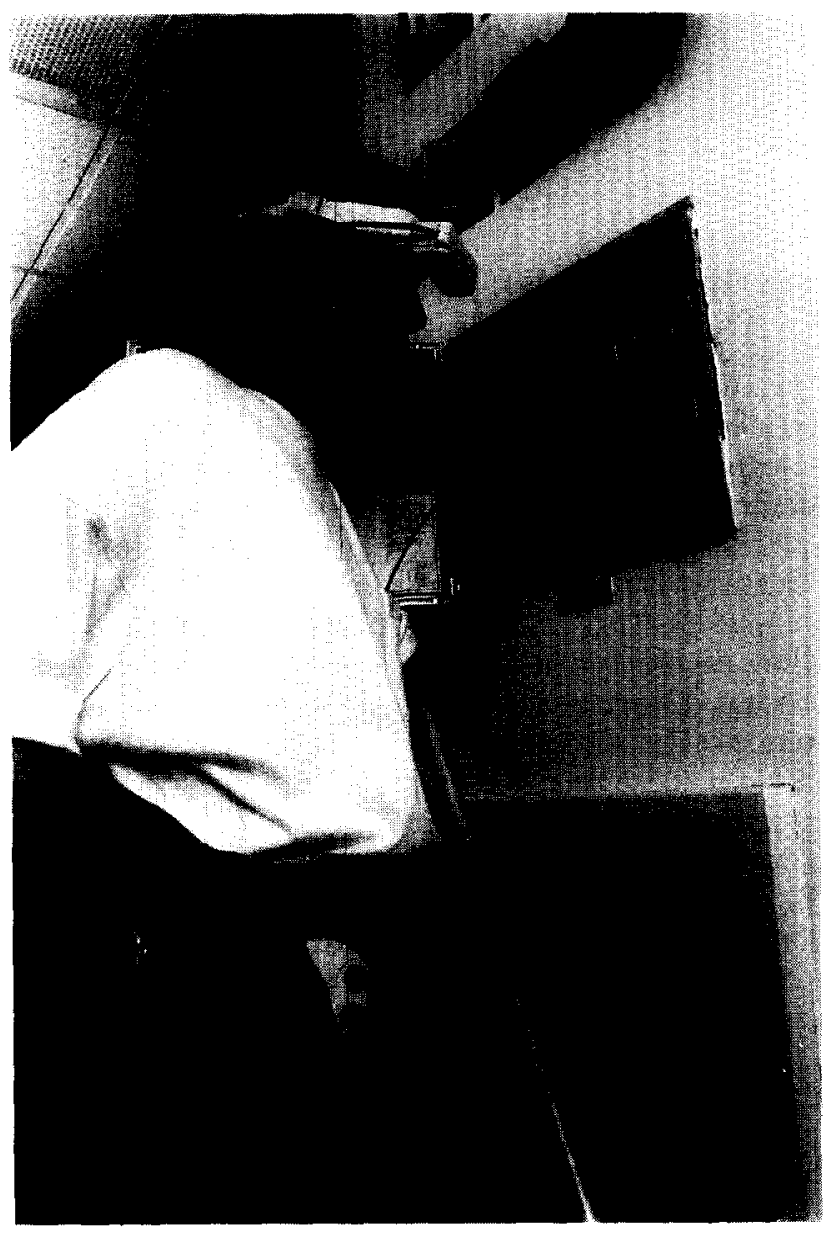

Figure 2 VuMan 2

Shape Deposition's primary attributes from a design point of view is that it removes traditional manufacturing constraints thereby significantly increasing the possibilities in form and function. Using a layered-deposition process provides access to all points on the interior

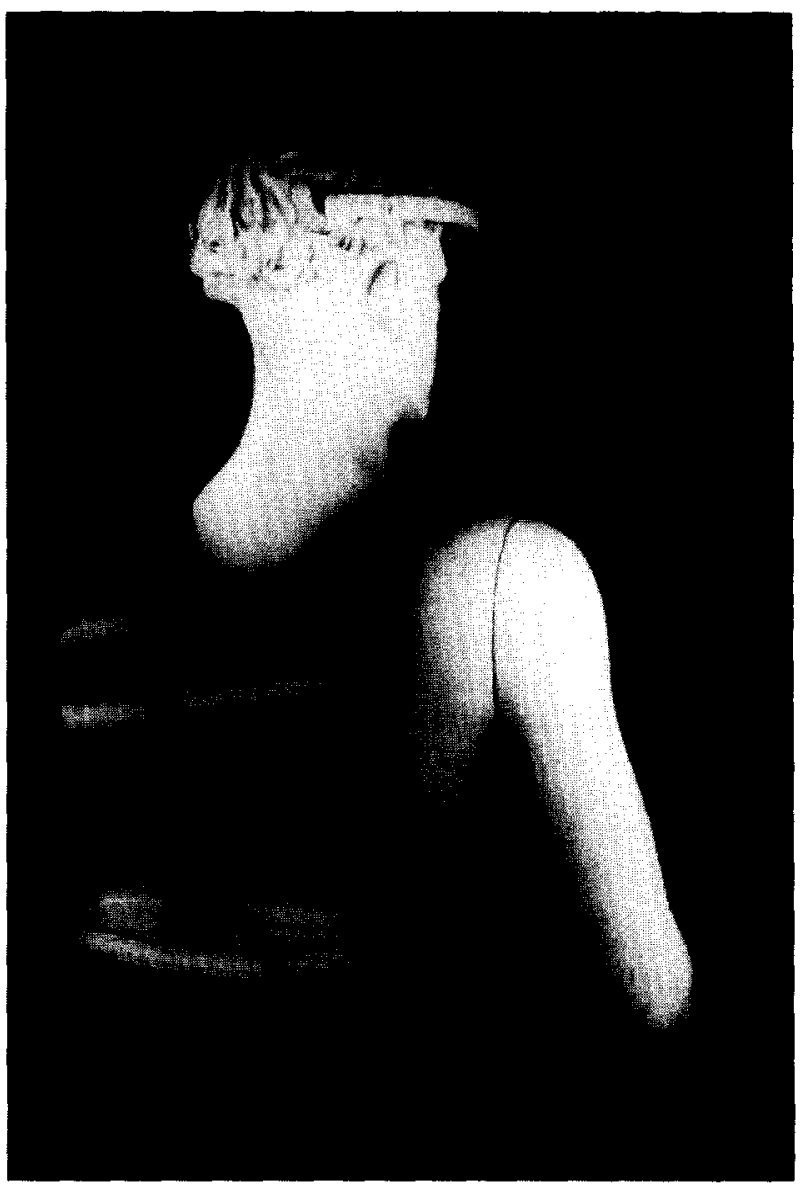

Figure 3 Navigator 1

of the part during manufacturing. Figure 5 shows a part on its pallet part way through the manufacturing process.

An important attribute of the Shape Deposition process, as well as other solid freeform manufacturing processes, is that it requires minimal fixturing and

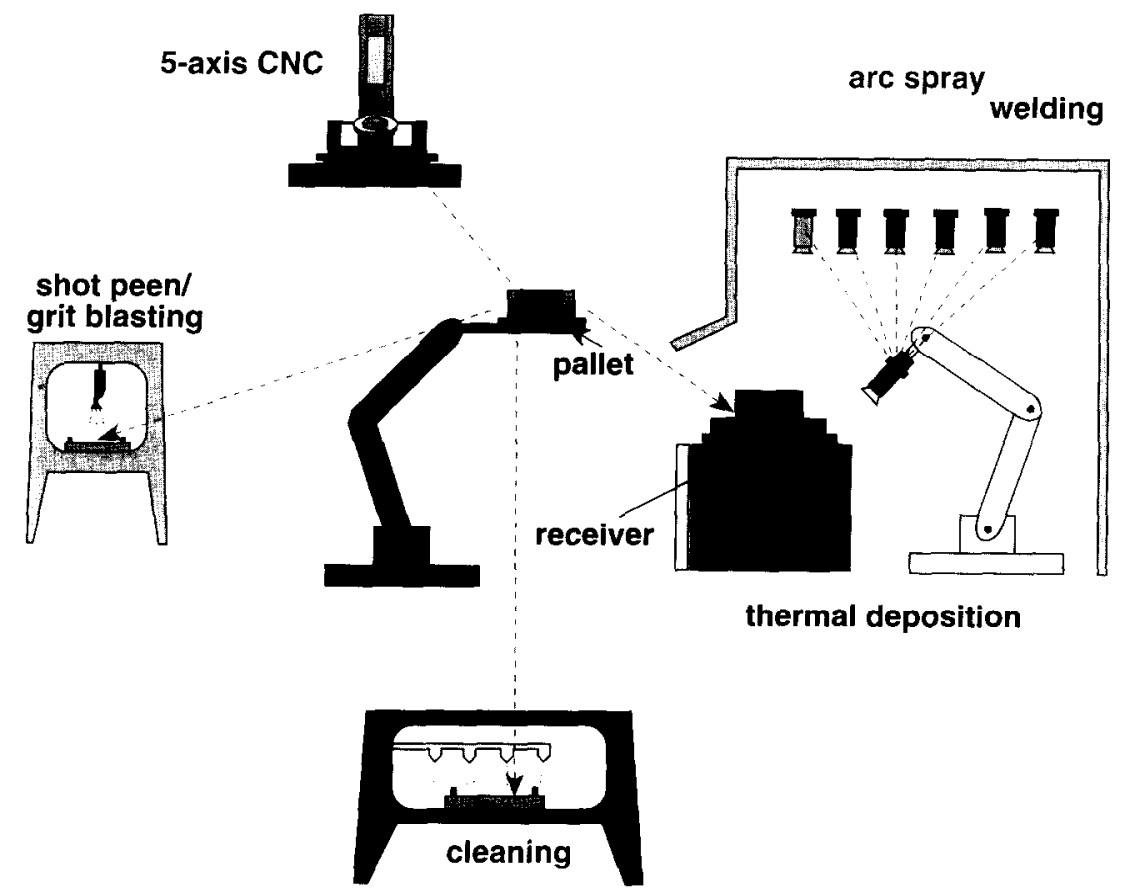

Figure 4 Shape deposition subprocesses to form a single layer shape deposition 


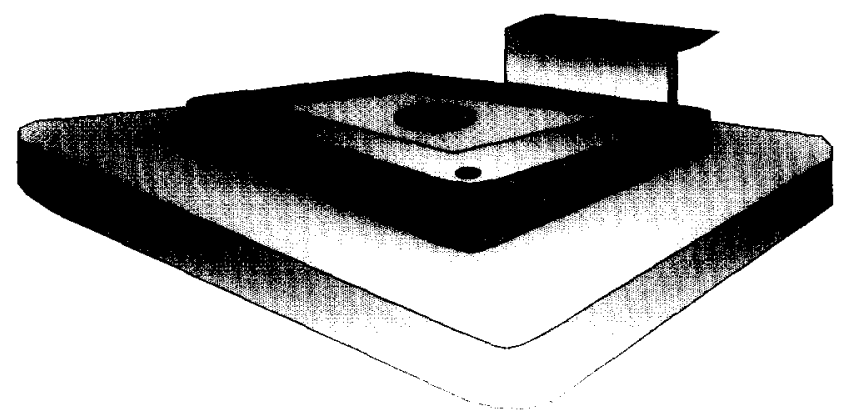

Figure 5 Cross-sectional layer of a sphere embedded in a cube

set-up. As Figure 5 illustrates, the sacrificial support material corresponds to the fixturing in traditional manufacturing processes. For Shape Deposition, process planning consists of filling the voids with support material, slicing the part into layers, and computing the tool paths to deposit the different materials in each layer. The process plan can be generated automatically from the CAD model, so parts can be sent directly from the design system to the manufacturing system.

Salient features of the Shape Deposition process are its ability to handle complex geometries, to vary shape and material composition continuously with the part, to fabricate assemblies in place, to embed electronic components, and to make electronic packaging an integral part of the mechanical structure. The Shape Deposition process allows encapsulation of prefabricated parts, such as computer chips and multichip components, by placing them in sockets and building the structure around them. Thus, electronic packaging becomes an integral part of the mechanical structure and electro-mechanical assemblies can be fabricated as a single structure.

During operation of embedded electronic components, the heat generated by the electronics must be removed. Using Shape Deposition, heat spreaders can be designed and manufactured as an integral part of the structure. Vias and other mechanical components can be embedded in the same manner as electronic components as illustrated in Figure 6. Figure 7 shows an electronic circuit that was embedded in a monolith manufactured using Shape Deposition. Figure 8 shows a Simon game manufactured using Shape Deposition, with the circuitry, lights, and switches embedded in a wrist watch-size structure.

\section{DESIGN PROCESS FOR THE NAVIGATOR}

Over the three semesters that the wearahle computers have been designed and manufactured, a design methodology has evolved that is used by the class. This methodology is described in more detail in Reference 3. This design methodology has its roots in electronic design, which has been the driving factor for wearable computers. The goal of the design methodology is to allow as much concurrency as possible in the design process. Concurrency is sought in both time and resources. The semester is divided into phases; activities within a phase proceed in parallel and are synchronized at phase boundarics. Resources consist of personncl, hardware platforms, and communications. Members of the design team are dynamically allocated to groups that focus on specific problems. Groups and individuals communicate informally between the synchronization points as well as formally during progress reviews.

The class draws students from all the departments affiliated with the Engineering Design Research Center. The following disciplines are necessary to design the Navigator:

- Electronics: electronic components, electronic interfacing, power supply;

- Industrial and mechanical: ergonomics, housing, component placement, thermal management, mechanical fixtures, design for manufacture, design for assembly, design for repair, design for recycling;

- Software: operating systems and support software. such as user interface tools, libraries for position sensing and telecommunications, hardware drivers; and

- Human-computer interaction (HCI): speech and user interface.

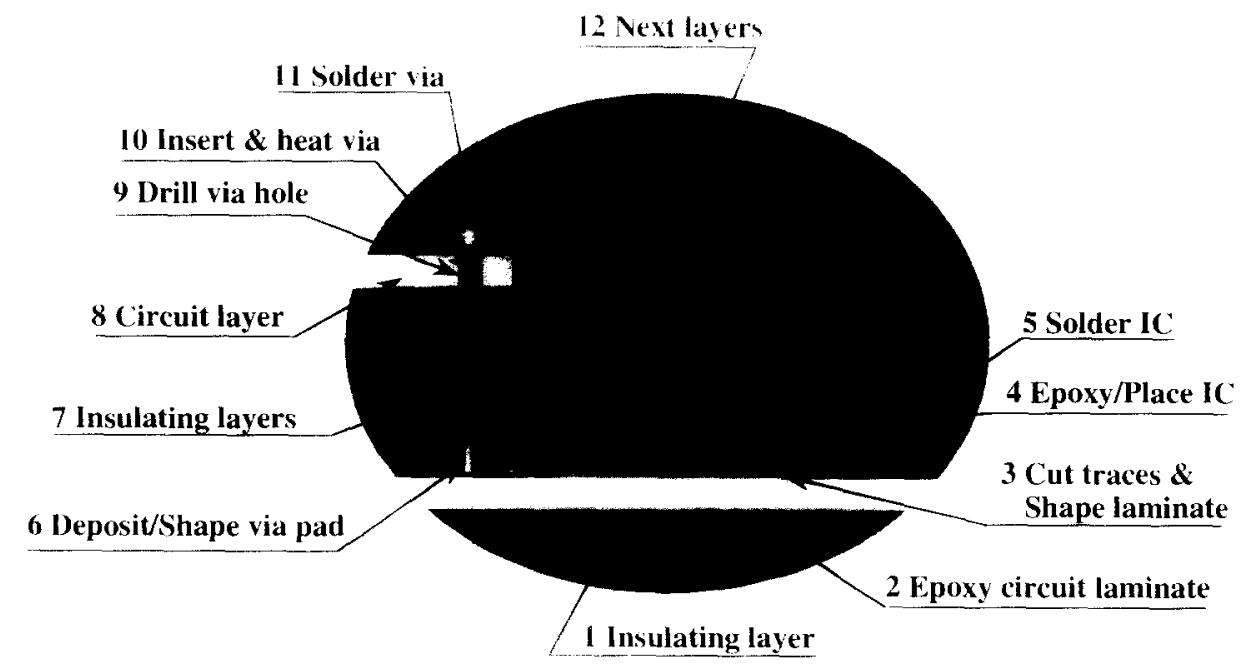

Figure 6 Embedding an electronic component and its couling structure 


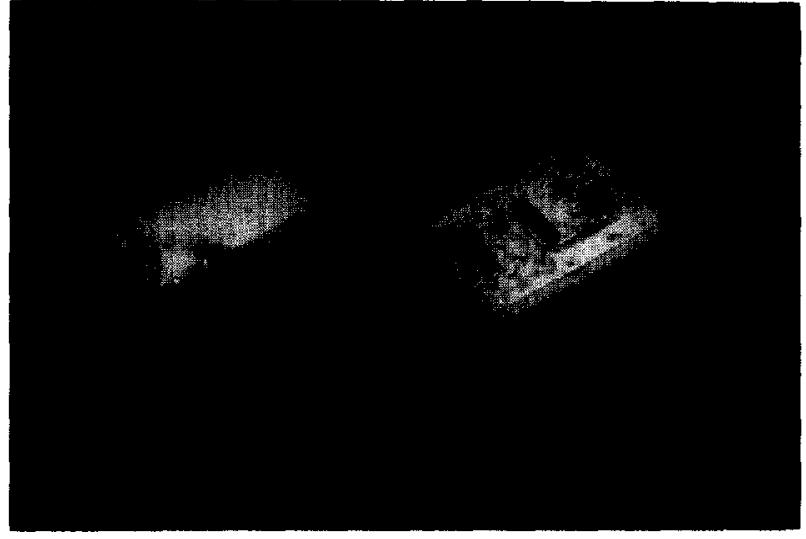

Figure 7 Electronic circuit embedded in a solid structure

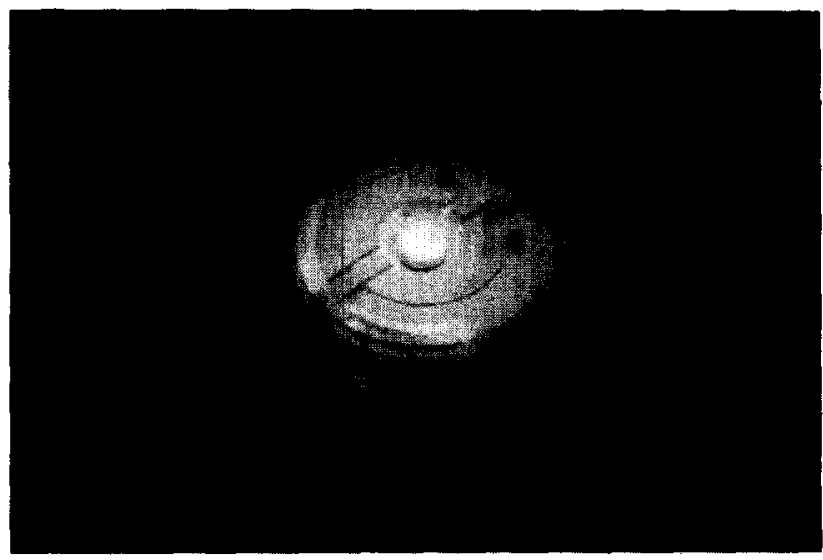

Figure 8 Wrist Simon game manufactured using shape deposition

The design process is broken down into six tasks:

(1) Technology suruey: Alternative technologies are identified for each functional requirement and evaluated in isolation from other requirements. Each group conducts a survey of available technologies. Alternative technologies are evaluated using Model Feature Matrices based on information gathered mostly from product literature. The groups identify primary and back up alternatives for each subsystem.

(2) System architecture specification: In this phase, the team produces several concepts of the total system by integrating the results of the tcchnology survey phase. The team identifies interactions and interfaces between subsystems and detects inconsistencies between subsystem alternatives. Interactions between subsystems are summarized in a Design Dependency Matrix. These dependencies identify communication points for subsequent phases of the project. The groups refine the primary technology alternatives for each subsystem to eliminate inconsistencies and to create a single concept. Based on the refined set of technology alternatives, the project team designs an architecture for the total system.

(3) Subsystem specification: The groups identify both firm and probable design decisions and produce specifications for each subsystem based on these design decisions. Each group must specify completely the interfaces to its subsystems so that other groups can continue into the detailed designed phase. Subsystem specifications are integrated into a complete Produce Design Specification. This phase represents the last exploratory phase of the design process.

(4) Detailed design: The groups perform a detailed design of each subsystem paying particular attention to maintaining the interface specifications as defined in the Product Design Specification. The groups acquire or build each subsystem and analyse it in terms of functionality and performance. Analysis of the technology may necessitate changes in the subsystem specifications, which must be communicated to the relevant groups. The team holds regular design reviews to ensure that interface specifications are not violated. Task Dependency Graphs are used to identify individual design decisions that affect more than one group in order to propagate design changes rapidly.

(5) Implementation: After detailed design is completed, each group implements their subsystem using the acquired technology. As implementation progresses, the groups demonstrate the subsystems at various stages of development to each other. The team uses a check list of open issues and action items to highlight remaining design dependencies and to schedule their resolution.

(6) System integration: The groups test each subsystem individually and then integrate them into the final system. An integration tree is used to sequence the merging of subsystems along the designed interfaces.

The communication patterns vary among groups with the design phases. Certain forms of communication are better suited to particular phases. The main forms of communication are:

- Group presentations: During group presentations, one group presents results to all members of the project design team. These results are in the form of design proposals, survey summaries, and demonstrations. Group presentations usually are the synchronization activity between time phases and allow for formal exchange of ideas between groups.

- Group meetings: Members of each group hold regular meetings to discuss issues related to their subsystem, e.g. brainstorming of subsystem designs, delegation of tasks etc.

- Progress reviews: The project leader monitors the progress in the design of the whole system. A check list of pending and completed milestones is created and updated during progress reviews. Progress reviews allow co-ordination among the groups during the later design phases when initial system integration commences.

- Electronic mail: Mailing lists are created for each group and also for the entire project team. The mail message pattern varies substantially during the different design phases. 


\section{CASE STUDY OF THE NAVIGATOR DESIGN}

Detailed case studies of the design of each generation of wearable computer have been published elsewhere ${ }^{1-3.5}$, so this section will cover the Navigator design only briefly.

The initial specifications for the Navigator were developed during meetings between the leaders of the project team and our sponsors. The functionality of VuMan 2 was the starting point of the discussions. The initial specifications of the Navigator included:

- Functionality;

- Hands-off operation:

- Speaker independent, continuous speech recognition;

- Capability to display text and graphics:

- Miniature; light-weight, head worn display;

- On board database of information and maps:

- Differential Global Position Sensing (GPS): and

- Modem/wireless communication with $k$. mote site.

- Performance

- Weigh less than $10 \mathrm{lbs:}$

- At least 2-h battery life-time before charging;

- Position sensing accurate to $5 \mathrm{~m}$ :

- 80-90\% accuracy in speech recognition:

- Screen refresh should not distract the user: and

- Cost less than $\$ 4500$ per unit.

As technological possibilities and limitations WCIL discovered, the initial specifications of the Navigator were refined and clarified. In addition, because the wearable computers are designed to assist users with their work, and because users wear the mobile computers, the Navigator needed to be evaluated in use by potential users. Without a physical object to interact with, it is difficult for users to tell whether the computer would meet their needs. So several prototypes were built and evaluated by the participants. The specifications evolved through these interactions between the participants and the prototypes.

During the conceptual stage, the multidisciplinary project design team established a common vision of the end product. This vision provided a consistent set of design goals for all disciplines to maintain throughout the product development cycle. Without a common understanding or vision between design groups and their members, each would be forced to rely on their own set of assumptions and criteria based on only a single view of the product.

A fundamental Navigator concept is modular design, in which each module performs a specific functional task. These tasks include information processing, visual display, speech recognition, position sensing, telecommunications and power. Each task may have several implementations, allowing the system to be more flexible to meet the needs of a specific application. Both physically and electronically, potential users of the Navigator system can tailor the unit according to application-specific needs. From the perspective of flexibility, the modular concept also allows the system to be updated or repaired simply by unplugging and replacing the appropriate module.

After reviewing the pre-existing mobile systems, the project team brainstormed alternatives for the Navigator wearable computer. They identified criteria for evaluating design alternatives, such as cost, weight, and development time. The target application was navigation around the Carnegie Mellon campus. To explore cach of the technology alternatives concurrently, the project team split into four subgroups: electronics (also known as the hardware group), mechanical and housing design, system software, and applications. Each group conducted an independent technology survey by contacting vendors, doing a literature survey, and consulting local experts. The findings in the Technology Survey were reported as Model Feature Matrices which listed and compared alternatives using the evaluation criteria identified initially. The groups presented the results of their Technology Survey phase and each team member received the written reports, including product specification sheets. The team as a whole discussed the results of the lechnology survey phase.

Each group presented the primary alternatives in detail during team meetings. The team identified subsystem interactions and dependencies and agreed on which design groups to notify if certain design decisions were made or modified. Compatible design alternatives were labelled resolved (i.e. the disk drive and the processor interfaced to the same bus, the operating system had a software device driver for the disk drive etc.), while other dependencies were labelled unresolved and required further refinement in the next phase.

The team members continued to keep each other informed about concerns that cut across groups. For example, during this stage, the thermal designer insured that all team members were cognizant of cooling issues. By doing so, the project team could focus on concepts that minimized heat production and could accommodate any necessary cooling strategies. The requirement for outdoor usage virtually mandated the Navigator to be a closed, ventless system. Therefore, heat dissipation needed to be kept as low as possible. The modular approach was advantageous from the thermal perspective, because it subdivided the total power output and increased the surface area through which heat could be dissipated. The desire for extended battery life established power minimization as a shared objective between the electronics and thermal designers

A new group formed to focus on speech recognition since this appeared to be the subsystem which was the least well understood and developed. The group selected Sphinx 1 as the primary alternative for speech recognition ${ }^{6}$ and Mach as the primary multitasking operating system ${ }^{7}$. Both selections were due to the availability of local experts. The Intel $80 \times 86$ processor family was the processor of choice for Mach. Because the 80386 had the highest performance and lowest power consumption of the available $80 \times 86$ chips at the time of the project, the group narrowed the design alternatives to low power consuming boards based on the 80386. The hardware and software groups worked closely together because their decisions were mutually dependent. The only satisfactory alternative for display was found to be the Private Eye ${ }^{8}$ and it became the 
default. The group could not resolve other dependencies without further study. The team discussed resolved and unresolved design alternatives during the group presentations.

Next, the project team produced an interface specification between subsystems so that the detailed design and implementation could proceed concurrently between groups. Subsystem specification is the last exploratory phase of the design and combines both bottom-up and top-down features. From the top down, the work on subsystems proceeded from the specification of the total system. From the bottom up, each subgroup provided a subsystem specification with special emphasis on how the subsystems interacted with the total system. The interfaces were specified in sufficient detail for other groups to continue into the next design phase.

As the design progressed, each group worked on a subsystem of the design; however, there were frequent interactions between the groups. For example, to allow concurrent hardware and housing development, a fullscale, nonfunctional prototype of the printed circuit board was fabricated. A ProEngineer model ${ }^{9}$ of the prototype provided detailed geometric information so a thermal analysis could be performed as the final check on the housing and the thermal design before building a functional prototype.

Because of the dependencies between computer chip selection, thermal analysis, and housing design, a thermal design methodology was proposed that used successive model refinement ${ }^{5,18}$. Surrogate models, which are statistical models that approximate more detailed physics-based, numerical models, were employed in the early design stages to evaluate alternatives and to reduce the number of designs to be considered. As the design evolved, successively more complete and accurate models were employed. The model evolution culminated with a conjugate conduction/convection direct numerical simulation on a Cray C-90 of the final design before it was manufactured.

During the implementation phase, the primary tool used by the team was an evolving list of open issues and action items. The final phase was to bring all the subsystems together to test and exercise their interfaces. The team used an integration tree to sequence the integration steps and set deadlines for the next step. Figure 3 shows the final system.

\section{THE NEXT GENERATION OF WEARABLE COMPUTER}

The generations of wearable computers have alternated between proofs of concept and refinement of technology. VuMan 1 demonstrated the functionality of a wearable computer; VuMan 2 provided twice the functionality, but at reduced weight, cost, power etc. The next VuMan will provide the same functionality as VuMan 2, but will be a proof of concept of the Shape Deposition Manufacturing process. The first Navigator, with global positioning, speech recognition, and telecommunications, provided an order of magnitude more functionality than either VuMan 1 or 2. The goal for the next Navigator is to reduce its size, weight, and cost.

Designing an electro-mechanical product to be man- ufactured on Shape Deposition Manufacturing requires tight integration of software, hardware, mechanical, and thermal design from the earliest stages of design. The relationship between hardware and software design will be similar to the relationship for Navigator; however, hardware design will be more constrained by mechanical and thermal requirements. The 3D arrangement of electronic components must satisfy constraints on electronic routing and timing, thermal interactions, structural integrity, in-place assembly, reliability, component accessibility for maintenance and replacement etc.

Many issues must be resolved for successful synthesis and analysis of conformal wearable computers with 3D electro-mechanical layout. One issue is the availability of appropriate computer-based tools. None of the constraints mentioned above arise in standard manufacturing processes, so each of these constraints will require the development of new tools. For example, the current tools for electronic placement and routing assume that the components will be laid out on $2 \mathrm{D}$ printed circuit boards. We need tools that allow 3D placement and routing of electronic components, but currently we do not have algorithms for this task. We also need algorithms that can transform the routing diagrams into $3 \mathrm{D}$ wires to be manufactured with Shape Deposition. A 3D geometric model that includes the electronic components and circuits is also required to perform an accurate thermal analysis.

Most of the available tools, both commercial and academic, do not share common representations and do not inter-operate effectively. Previously, we have used a blackboard architecture ${ }^{11}$ to address this problem ${ }^{12}$; however, blackboard architectures assume a shared computational space (the blackboard), a shared product and process representation, and a well-defined control strategy. When the design tools, the design process, and the manufacturing process are all changing rapidly, blackboard systems are not flexible enough. In addition, creating a shared space is difficult when the design tools and the design team members are distributed over a computer network. No synthesis tools account for the simultaneous constraints that arise from different domains and that must be resolved in the final product. Inter-operability of computer tools, as well as communication of knowledge among team members, will be major issues for designing the next generation of wearable computers.

Finally, with each generation of wearable computer, we have seen the demand for decreased product development times and increased product functionality. Under these conditions, the ability to make rapid prototypes - no matter what the medium - is essential. Getting the right product out the door on time cannot be achieved just by getting the technology right; we must also produce the right product. Getting the right product requires feedback from all the participants including team members, sponsors, and users throughout the design process.

\section{REFLECTIONS ON THE DESIGN PROCESS}

Just as product design is an iterative process, so is process design. With each new cycle, we gain experience and knowledge about the products we have de- 
signed and about the processes we use to design them. Design must be an evolutionary, responsive process that accommodates new experiences, knowledge, and situations. Reflection and change are necessary to maintain a vital process.

As educators and researchers. we have many customers and responsibilities; our customers are our undergraduate and graduate students, our government and industry sponsors, the end users of the tools and products we create, as well as society at large. Each of these is a participant in the design process whether directly or by proxy. Due to the multiple, diverse participants, each design project will have mismatches of goals and desires. Bringing all the participants actively into the design process at the early stages to develop it common vision can help to articulate and resolve por tential conflicts before they arise.

Time is a resource that universities have that industry does not; we have time to research and to experiment without the pressures of market constraints and high risk investments. At the EDRC, we have created a design research laboratory in which we nol only cieale products, but also address issues in product innovation and evolution, in design process and methodology, and in curriculum development. We have created an environment in which the designers are participants in the entire process, with the power to try something on the side, in which failure is not punished, but considered part of the design process as well as part of the learning process, and in which designers have time to reflect on their experiences.

\section{Conceptual design}

The conceptual phase proceeds from problem definition to product definition. In this phase, the team identifies the needs of participants and begins to translate those needs into product attributes. The needs provide the basis for focusing concept generation and design activities. The criteria developed during the problem definition has served as the basis for bench marking competitive products, past projects, projects in progress and early prototypes.

The inherent value of a product must be measured across its entire life-cycle. As designers, we must attempt to satisfy the needs of all the participants in the product's life-cycle. The activities in a product's lifecycle may include: planning, marketing, development, engineering design, manufacturing, distribution, sales. installation, training, novice use, expert use, customer service, repair, refurbishment, resale, and finally recycle or disposal. Designers must address issues related specifically to each activity and must address the concerns of the participants involved within the activities including their physical, cognitive, and emotional needs.

An integrated product definition process is required to understand the design, technical, and business implications of product choices as early in the design process as possible. When considering tradeoffs, the designer needs to know the relationships among a product's attributes, for example, between cost and quality, weight and durability, or performance and reliability. Concepts such as the house of quality ${ }^{13}$ and total quality loss ${ }^{14}$ can be used to structure these tradeoffs. Understand- ing tradeoffs focuses a team's efforts on those product attributes most directly related to improving user satisfaction. For example, does increasing the computer clock speed have a negative effect on reliability and which is of greater concern to users? By assigning target values and understanding these qualities, the ream can set priorities for product specifications. These criteria provide the basis for choosing a design direction and for forming an implementation strategy. It is imperative that these criteria and strategies be revisited, revised, refined, and redefined throughout the process.

The most important benefit of applying an integrated product definition process is that the design can be redirected early, before expensive design activities have started and before time is wasted developing the wrong product. In most cases, we have found that explicitly considering the product life-cycle early in the design process results in significant improvements in the form and function of the design as well as in the users perception of the quality of the design.

Oui methodology as it stands emplasizes the design phases of the product design cycle. However, the initial conceptual and planning phases drive all the decisions afterward. If not done thoroughly with all team members involved, later in the process the product begins to resemble a jumble of force-fit components rather than an integrated design that successfully meets the participants" needs. At the EDRC, we need to expand the explorations of our conceptual and planning phases. The potential of our projects, and expectations, consistently has depended on the decisions made in these carlier phases; decisions that many times need to be refined or even redefined as the project matures.

\section{Design process planning}

Product design is not a simple process that progresses linearly from idea to reality; it is an extensive process that requires resources and planning. During the planning phase, the team begins to understand the interactions and interfaces necessary for the project to succeed. During planning, team members define the key events that will facilitate the design process.

Planning begins within the conceptual phases of the project, where documentation includes: a problem statement, technological alternatives, market analysis and planning, user research, visualization and product concepts. Within the activity of planning, we are beginning to cstablish an approach that anticipates the dcsign process tradeoffs that inevitably occur. Because of the scope of our projects and the exploratory and experimental approach of the EDRC, we need our methodology to accommodate flexible schedules, allowing them to change throughout the project to better match the emerging needs of the project team.

\section{Teamwork and communication}

Design is a social process, in which designers interact with one another, with sponsors, with users and with other participants in the process in order to identify a need, define and redefine the problem, specify and refine a solution, and communicate to others how to 
realize the solution. Designers spend only about $10-15 \%$ of their time on discipline-specific tasks ${ }^{15}$, the rest of their time is spent talking, writing, negotiating, meeting, searching, documenting etc. Information exchange needs to be a guided activity - not just an activity that designers are told they should do without guidance or encouragement.

Interdisciplinary groups collaborating on a design project do not necessarily make a team. People need practice and time to develop trust and to develop a working relationship. Designers need to understand and appreciate how different disciplines approach problems, how they talk about problems and solutions, and what tools they use to solve problems. The unpredictable interactions that occur between designers and between disciplines are essential in interdisciplinary design. The solution to concurrent design is not connecting discipline-specific computer-based tools together, it is creating an interdisciplinary team of people who can work together, aided by tools that support the design process.

Within our design process, we include activities to help students learn to work in teams, to explore the dynamics of the team and to understand the strengths, weaknesses, and roles and responsibilities of individuals and groups within the project team.

\section{Rapid prototyping}

Designers alternate between the abstract and the concrete ${ }^{16}$; that is, a team's first ideas are turned into rough sketches, these sketches are evaluated, new ideas emerge, and more precise drawings are generated. This iterative process continues with soft mock-ups, appearance sketches, computer and shop prototypes, until finally the product is fabricated. An important part of this process is the evaluation of the prototypes. Prototypes can help to redefine, evaluate, and analyse the participants, needs and requirements. Rapid prototyping can reduce the time and increase the quality of the iterations in the design cycle. Currently, we use prototypes to evaluate the designs as they evolve. We do not formally use the principle of spiral development, in which the design problem and its solution evolve in parallel $^{17}$, but the idea of participatory design has influenced our methodology.

\section{Analytical tools}

Although the availability of inexpensive, fast computers permits numerical prediction of complex phenomena not previously possible ${ }^{18}$, the analysis of many phenomena remains intractable in a design environment. This is especially true in concurrent design because of the stringent time constraints associated with the early design stages wherein large-scale simulations are too resource intensive and time consuming ${ }^{19}$. In particular, the large design space associated with initial design stages prohibits detailed simulations of all permutations of a design. For some critical aspects of wearable computers, such as thermal analysis, we have developed analytical tools that let the designer study the effects of design decisions in the preliminary, detailed, and final stages of design 5 .

An added difficulty is that we are designing novel products and, with the next gencration, we will be using a novel manufacturing process. In both cases, the novelty reduces the number of commercial tools that are applicable within our design process. The novelty also increases the number of doctoral students working in areas related to the wearable computers. Unfortunately, due to the long lead-time of doctoral theses and to the brittleness of most tools written as part of doctoral theses, their tools are difficult to integrate into the design process. So, with each generation of wearable computers, fewer computer-based tools have been used.

For some analysis tasks, commercial tools have proven effective because they have better user interfaces and more functionality than the research prototypes that are created in a university setting. These tools have helped reduce the number of iterations between design and manufacture, increase the accuracy of the components, and increase the accuracy of the interpretation of design specifications. However, commercial tools are difficult to integrate into a larger system because each tool is designed to be used alone. Integrating computer-based tools has many of the same problems as creating design teams. Each tool comes with domain-dependent jargon and assumptions. The inability to share knowledge and information between tools is a large barrier to integrated design. Research is just beginning to address this issue ${ }^{20}$.

In a concurrent design framework, domain-specific analysis models require modifications to:

- account for constraints imposed by other domains,

- maintain consistence across design views,

- allow for rapid incremental redesign.

\section{Distributed design}

The research at the EDRC and elsewhere in academia tends to focus on the analytical and technical aspects of the design process. However, communication and inter-operation are essential to concurrent design. We must begin to look at tools that support relationships rather than analytical tasks. As part of another research project, we have begun to work on an open network that will allow people to collaborate and share tools over the Internet ${ }^{21}$. We propose to use the wearable computer class as a testbed for some of the initial experiments to discover how teams can collaborate when not all the team members are in the same location.

\section{Design history and documentation}

One of the clear lessons from our experiences with designing the wearable computers is that we must pay more attention to history - both our own and the world's. Users' needs have connections and relationships with their experiences, conventions and culture. The technology survey phase, in which we look at the 
artifacts that already exist, is based on the premise that all design is redesign.

One aspect of tracking history involves automatic capture of the communication and design dependencies. The automatic capture of the information that forms the shared meaning for a team is the goal of $n$-dim, a system that is under development at the EDRC $^{22}$. n-dim is based upon two observations about design, observations that are supported by our experiences in the wearable computer design projects:

- Progress in design is made by creating and refining a shared meaning of the requirements and of the potential solutions through negotiations, discussions, clarifications and evaluations.

- Design efficiency is achieved through integration of information acquired from a variety of multidisciplinary sources.

We must look at our own history. By analysing our own history, we can discover and cvaluate why we missed deadlines, why we were successful, what we missed in product value opportunities etc. The knowledge gained in this process can help guide the next project and help the continual evolution of the design process. This reflection can also provide new teams with examples from the past, giving them an experience that is not yet theirs to help them anticipate interactions and interfaces. The history provides a point of reference and assists in making team members aware of the scope and reality of the project, especially for people who have worked only in the context of their own discipline, but who do not understand how their work fits into the whole, their relationship to the team. or the languages of their team members.

\section{Fducation}

We learn from the mistakes we make while gaining experience; we gain experience from experimentation; we learn through our conversations in speech, movement, sketches, and text; and we build our knowledge through conversation, experimentation, and reflection. The student design experiences should not be only about the success of the final product, but about learn. ing to experiment, fail, converse, share, reflect and create. Learning these skills may require unlearning some of the skills acquired in a standard undergraduate engineering education in which sharing and failing are discouraged and in which the skills of conversing and reflecting are often considered unimportant.

We need to instill these ideas in our students, so they do not proceed on the premise that they simply need to get the job done. The most important aspect of the student design experience is the practice of reflection, a practice many of us bypass because of time constraints. Within a university setting, we work in a reduced form with a more manageable environment in which we can take the time to teach our students the skills they need.

We need to encourage an atmosphere and process that invites ownership and pride in the completeness and professional quality of the work. We also need to encourage an atmosphere in which students can have fun designing novel artifacts.

\section{CONCLUSION AND FUTURE WORK}

We have designed and manufactured three generations of wearable computers and are starting on a new generation. The computers are designed and built by an interdisciplinary design team composed of students, staff, and faculty. Over the semesters that the class has been taught, we have developed an interdisciplinary concurrent design methodology that is constantly revisited and revised as new artifacts and processes come into play.

In future generations, we plan to use a novel layered manufacturing process, Shape Deposition Manufacturing and to have a design team where not all members are in the same location. Both of these plans will require changes, some fundamental, in our current design methodology. Using the Shape Deposition Manufacturing process will require even tighter integration between the different groups in the design team. Distributing the design team will require a re-examination of how the groups share the current context of the design and how decisions are communicated among the members of the groups and teams.

The engineering product design process has changed substantially over the last decade. It is moving from sequential to concurrent, hierarchical to parallel, deferred problem resolution to real-time problem resolution, paper data exchange to electronic data exchange, stand-along tools to integrated tools, limited design space exploration to comprehensive design space exploration. Design research continues to play a key role in successful experimentation within this changing environment.

\section{ACKNOWLEDGEMENTS}

This work has been supported by the Engineering Design Research Center, an NSF Engineering Research Center and by the Advanced Research Projects Agency. We would also like to acknowledge the work of the students in our classes who have made the wearable computers possible. Many graduate students and research staff have also contributed to the success of the project, among them are: Drew Anderson, Kevin Hartman, Jay Nigen, Kevin Schmaltz, and Larry Schultz.

\section{REFERENCES}

Akella, J, Dutoit, A and Siewiorek, D P 'Concurrent engincering: a prototyping case study' 1992 Int. Wkshp. Rapid System Prototyping Research Triangle Park, NC (Jun 1992) pp 138-160 Smailagic, A and Siewiorek, D P 'A case study in embedded-syslem design: the VuMan 2 wearable computer' IEEE Des. Test Comput. (Sep 1993) pp 56-67

Siewiorek, D P, Smailagic, A, Lee, J C Y and Tabatabai, A R A An interdisciplinary concurrent design methodology applied to the Navigator wearable computer system' J. Comput. Software Engng Vol 2 No 2 (1994)

+ Hartmann, K. Krishnan, R. Merz. R, Neplotnik, G. Prinz. F B. Schultz, L, Terk, M and Weiss, L E 'Robot-assited Shape Deposition Manufacturing' Proc. 1994 IEEE Int. Conf. Robotics and Automation San Diego (May 1994)

Amon. C H, Nigen, J S, Sierwiorek, D P. Smailagic, A and Stivoric, $J$ 'Concurrent design and analysis of the Navigator 
wearable computer system: the thermal perspective' IEEE Trans. Components, Packaging, Mfg. Tech. (Dec 1994) pp 133-142

6 Lee, K-F, Hon, H-W and Reddy, R 'An overview of the Sphinx speech recognition system' IEEE Trans. Acoustics, Speech Signal Proc. Vol 38 No 1 (1990) pp 35-45

7 Rashid, R, Julin, D, Orr, D, Sanzi, R, Baron, R, Forin, A, Golub, $\mathrm{D}$ and Jones, $\mathrm{M}$ 'MACH: a system software kernel' COMPCON Spring ' 89 San Francisco (Mar 1989) pp 176-178

8 Becker, A 'Design case study: private eye' Info. Display Vol 6 No 3 (1990) pp 8-11

9 Parametric Technology Corporation 'ProEngineer release 8.0 ' Technical Report FPI-0991-02 Parametric Technology Corporation, Waltham, MA (1992)

10 Nigen, J S and Almon, $\mathrm{C} \mathrm{H}$ 'Concurrent thermal design: balancing accuracy with time constraints' IEEE Trans. Components, Hybrids, Mfg. Tech. Vol 15 No 5 (1992) pp 850-859

11 Erman, L D, Hayes-Roth, F, Lesser, V R and Reddy, D R 'The Hearsay-II speech understanding system: integrating knowledge to resolve uncertainty' Comput. Surv. Vol 12 No 2 (1980) pp 213-253

12 Finger, S, Fox, M S, Prinz, F B and Rinderle, J R 'Concurrent design' Appl. Artif. Intell. Vol 6 (1992) pp 257-283

13 Hauser, J R and Clausing, D 'The house of quality' Haruard Bus. Rev. Vol 66 No 3 (May 1988) pp 63-73

14 Taguchi, G and Clausing, D 'Robust quality' Harvard Bus. Rev No 1 (1990) pp 65-75

15 Bucciarelli, L L 'Reflective practice in engineering design' Des. Stud. Vol 5 No 3 (1984) pp 185-190

16 Adelson, B 'Comparing natural and abstract categories: a case study from computer science' Cognitive Sci. Vol 9 No 4 (1985) pp 417-430

17 Roozenburg, $\mathrm{N}$ and Cross, $\mathrm{N}$ 'Models of the design process integrating across the disciplines' Proc. ICED '91 Zurich (Aug 1991) pn 185-193

18 Amon, C H, Herman, C V, Majumdar, D, Mayinger, F, Mikic, B B and Sekulic, D P 'Numerical and experimental studies of self-sustained oscillatory flows in communicating channels' Int J. Heat Mass Transfer Vol 35 No 11 (1992) pp 3115-3129

19 Yesilyurt, S and Patera, A T 'Surrogates for numerical simulations; optimization of eddy-promoter heat exchangers' Comput. Meth. Appl. Mech. Eng. Vol 121 (1995) pp 231-257

20 Cutkosky, M R, Engelmore, R S, Fikes, R E, Genesreth, M R Gruber, T R, Mark, W S, Tenenbaum, J M and Weber, J C 'PACT, an experiment in integrating concurrent engineering systems' Computer Vol 26 No 1 (1993) pp 28-37

21 Coyne, R, Finger, S, Konda, S, Prinz, F B, Siewiorek, D P, Subrahmanian, E, Tenenbaum, M J, Weber, J, Cutkosky, M Leifer, L, Bajcsy, R, Koivunen, V and Birmingham, W 'Creating an advanced collaborative open resource network' Proc. Sixth Int. ASME Conf. Design Theory and Methodology Minneapolis MN (Sep 1994) pp 375-380

22 Levy, S, Subrahmanian, E, Konda, S, Coyne, R, Westerberg, A and Reich, $\mathrm{Y}$ 'An overview of $n$-dim environment' EDRC Technical Report 05-65-93 Carnegie Mellon University, Pittsburgh, PA (1993)

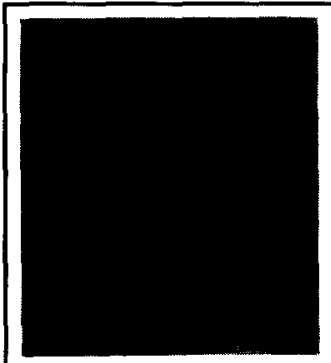

Susan Finger is on the faculty of the Civil Engineering Department at Camegie Mellon University. She is also affiliated with the Engineering Design Research Center, the Robotics Institute, and the Department of Mechanical Engineering. Dr Finger received her $B A$ in astronomy from the University of Pennsyluania, her MA in operations research from the Unitersity of Pennsylluania, and her PhD in electric power systems through Civil Engineering from the Massachusetts Institute of Technology. She was on the faculty in Manufacturing Engineering at Boston University and was a visiting assistant professor in Mechanical Engineering in the Laboratony for Manufacturing Productivity at MIT. She served at the National Science Foundation as the program director for Design Theory and Methodology. She is a founder and co-editor-in-chief of the joumal Research in Engineering Design. Dr Finger's research interests include representation languages for designs and integration of design and manufacturing concems.

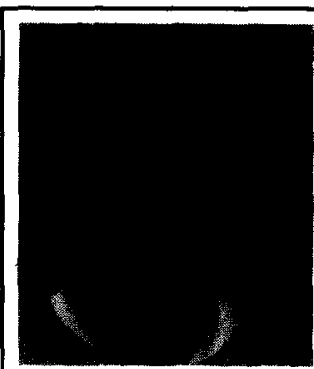

John Stivoric received his BFA in indus trial design, with unicersity and college honours, from Camegie Mellon University in 1993. He is currently working towards an MS degree in product design. $\mathrm{He}$ is an industrial designer with the Engineering Design Research Center at Carnegie Mellon University. His current work centres on interpreting and transferring advanced technologies, product development methodologies, and prototyping methods from the laboratory to industry, within an interdisciplinary design encironment. Mr Stivoric is also an instructor with both the Engineering. Design Research Center and the Department of Design at Carnegie Mellon University. He is a member and education chair of the Industrial Designers Society of America, Western Pennsyluania Chapter. He is also a member of the Human Factors Society.

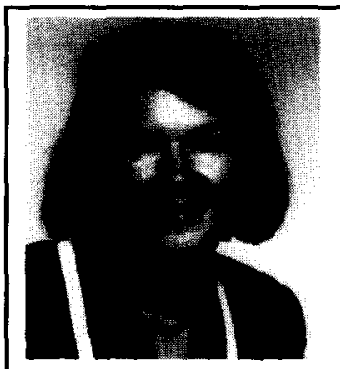

Cristina Amon received $M S$ and $S c D$ degrees in mechanical engineering from the Massachusetts Institute of Tech nology in 1985 and 1988, respectively. She joined the faculty of Camegie Mellon Unilersity in 1988, where she is currently an associate professor with the Department of Mechanical Engineering. Her research interest include computational fluid dynamics and heat transfer, flow stability and transition to turbulence, thermal management of electronic systems and wearable computers, electronic packaging, concurrent thermal design, heat transfer augmentation techniques, and thermal phenomena in spray shape deposition and microcasting manufacturing processes. Dr Amon received the NSF Initiation Award in 1989, the GT Ladd Award for Excellence in Research in 1991 and the SAE Teetor Educational Award in 1994. She is a member of the ASME Technical Committees on Heat Transfer in Electronic Equipment Fundamentals of Heat Transfer and Computational Heat Transfer.
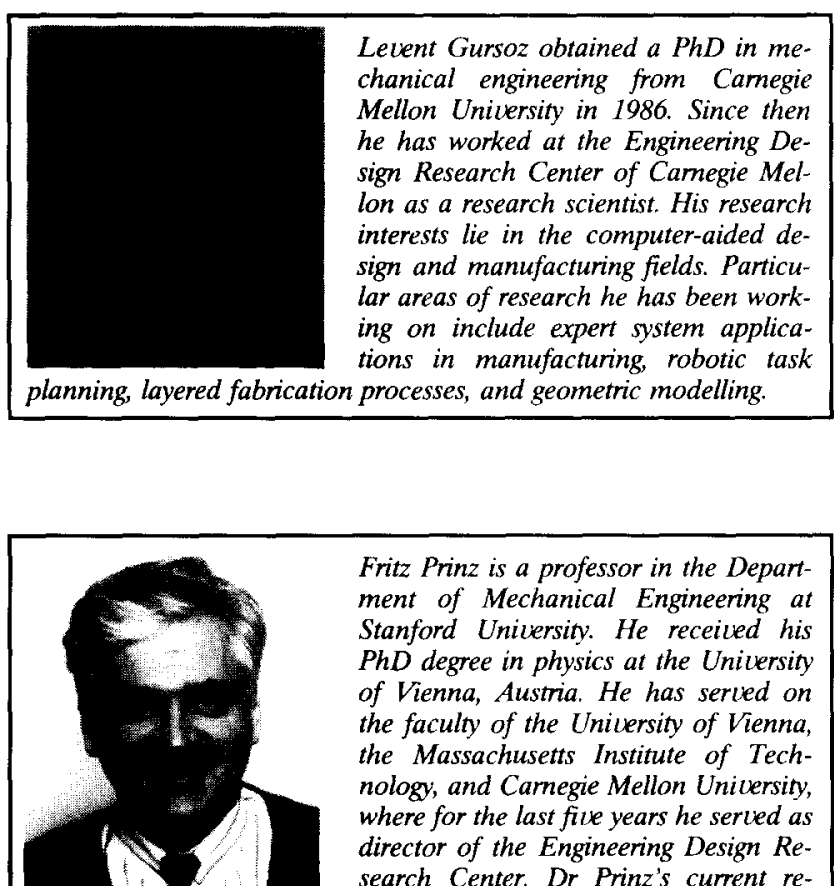

Fritz Prinz is a professor in the Department of Mechanical Engineering at Stanford University. He received his $P h D$ degree in physics at the University of Vienna, Austria. He has seried on the faculty of the Unicersity of Vienna, the Massachusetts Institute of Technology, and Camegie Mellon Unitversity where for the last five years he served as director of the Engineering Design Research Center. Dr Prinz's current research activities address a large area of problems related to intelligent design and manufacture. 


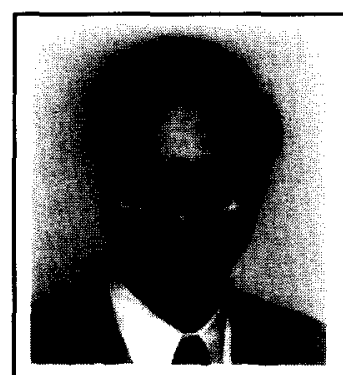

Daniel Siewiorek received a BS degree in electrical engineering from the Unilersity of Michigan, Ann Arbor, and MS and $P h D$ degrees in electrical engineering from Stanford University. He is a professor in the School of Computer Science and the Department of Electrical and Computer Engineering at Carnegie Mellon and is currently the director of the Engineering Design Research Center. Dr Siewiorek has published over 250 technical papers and 6 books, including The Theory and Practice of Reliable System Design. He was elected an IEEt Fellow for contributions to the design of modular computing systems' and has serced as chairman of the IEEE Technical Committee on Fault-Tolerant Computing. He was also awarded the Frederick Emmons Terman Award by the American Socity for Engineering Education for Olutstanding Young Electrical Engineering Educator and he receiled the Eckert-Mauchly Award gicen jointly hy the ACM and IEEE Computer Society for his outstanding contribu tions to the field of computer architecture.

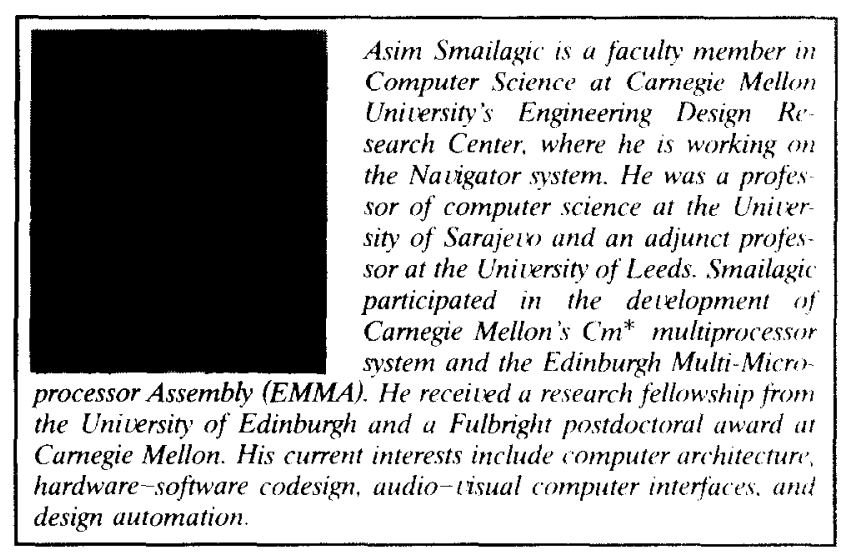

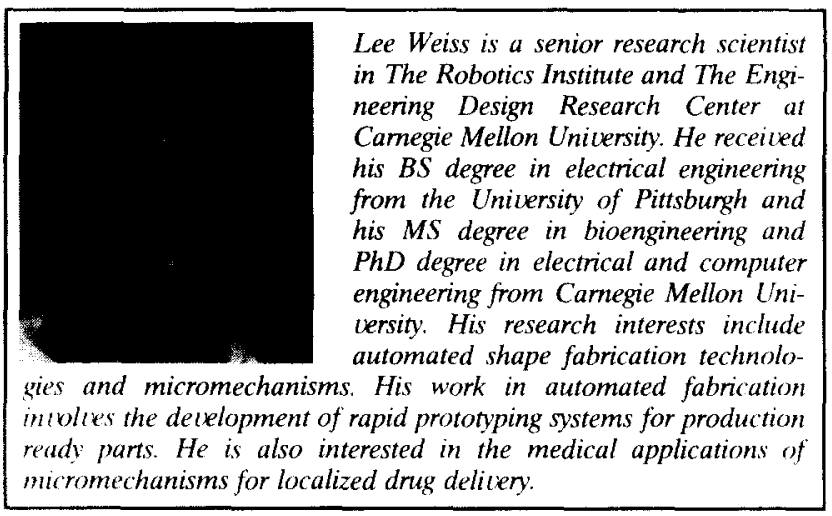

Lee Weiss is a senior research scientist Re Engi(nesearch Center ar armegie Mellon Unicersity. He receiled egree in electrical engineerin his MS degree in bioengineering and degree in electrical and computer ering from Carnegie Mellon Uniansity. His research interests include utomated shape fabrication technoloechanisms His work in automated fabrication ready parts. He is also interested in the medical applications of micromechanisms for localized drug delicery. 